\section{The National Junior Horticultural Association Photography Contest}

The National Junior Horticultural Association (NJHA) was established in 1935 to develop the horticultural expertise, skills, knowledge, and leadership skills of youth in the United States. NJHA organizes projects, contests, workshops, and other activities for youth at its annual convention (Fountain, 1991, 1992), rather than a program where youth advance from one project to another (Table 1).

Imaging techniques have long been an important medium for conveying horticultural knowledge. When Johannes Gutenberg (circa 1400-68) invented the movable printing press, individuals interested in plants were finally able to reproduce and disseminate rapidly and efficiently the drawings that accompanied botanical descriptions. This invention aided taxonomists such as Carl von Linne (Carolus Linnaeus, 1707-78) in classifying the tremendous influx of new species from the New World and Asia. Plant identification would have been considerably more difficult without these prints. The drawings helped eliminate duplicate names of countless species.

It was only a matter of time before the superior quality of black-and-white and, later, color photographs eliminated line drawings, except where simple illustrations were desired. Now, advanced imaging techniques such as aerial infrared photography, videography, and computer enhancement are essential tools for studies as diverse as urban planning, managing habitats, estimating crop yields, and documenting environmental degradation.

Video technology has advanced more rapidly than any other form of imaging technology and offers many instructional advantages over still photography. Even so, color prints and transparencies (slides) are essential for horticultural instruction through retail displays, books, and lectures. No technology is as versatile, efficient, cost effective, and easy to use. A sequence of photographs clearly and concisely illustrates the multiseasonal characteristics of a species. Few technologies have aided horticultural science as still photography has. While still-photography technology is destined to change, it remains an important tool for plant science.

\section{Skill development}

Photography is a tool used effectively by youth organizations such as NJHA, 4-H, and Boy and Girl Scouts of America. While the art and science of photography can be an end in

Received for publication 8 Nov. 1993.

'Associate Extension Professor, NJHA Program Chair. itself, it is a skill that brings a lifetime of personal pleasure and professional benefits.

Developing trust and honesty between adult leaders and youth are fundamental principles in most youth organizations. As a result, it is often necessary for adult leaders to accept the youth's word that something has been accomplished as described. Photographs help them document their progress and offer undisputed proof of their accomplishments.

As a tool, photography introduces youth to artistic principles and the wonder of nature. It teaches patience. Anyone who has ever waited with camera in hand for a bee to alight on a flower knows patience. Taking quality photographs requires planning so that lighting and shadows provide the right effect. Photography teaches an appreciation for balance and neatness. Creativity is encouraged in a world that seems prone to sameness. By photographing subjects in nature, it is possible for youth to develop observation skills, such as the exquisite detail of the simplest flower.

Through photography, youth develop an appreciation for artistic principles, develop self esteem, communication skills, and often become motivated to learn more about the subject of their photographs. Many youth become interested in other NJHA programs and activities as a result of photography.

\section{Photography contest}

Eight years ago NJHA created a contest for youth to exhibit photographs depicting horticultural subjects and activities. Photographs are judged and exhibited at the annual convention. The NJHA Horticultural Photography Contest consists of competition in three divisions: single black and white, single color, and four sequential photographs (either black and white, or color).

Entries in all three divisions are judged on quality (35 points), composition (35 points), and storytelling ability (30 points). Quality consists of focus, lighting/shadows, depth of field, distance from the subject, and neatness of the display. Composition incorporates originality, arrangement, and background. Storytelling must occur without captions or other verbal descriptions. This is especially important for those entering a sequence of four photographs. The winning entry must clearly demonstrate the old adage that "a picture is worth a thousand words." A classic four-photograph illustration begins with a photograph of seed and ends with a photograph of a finished, salable horticultural product. The story must relate to horticultural products or show the progression of a horticultural activity. The evaluation team consists of a professional horticulturist, a professional photographer, and a youth officer from NJHA to ensure balanced evaluations.
Table 1. Projects sponsored by NJHA.

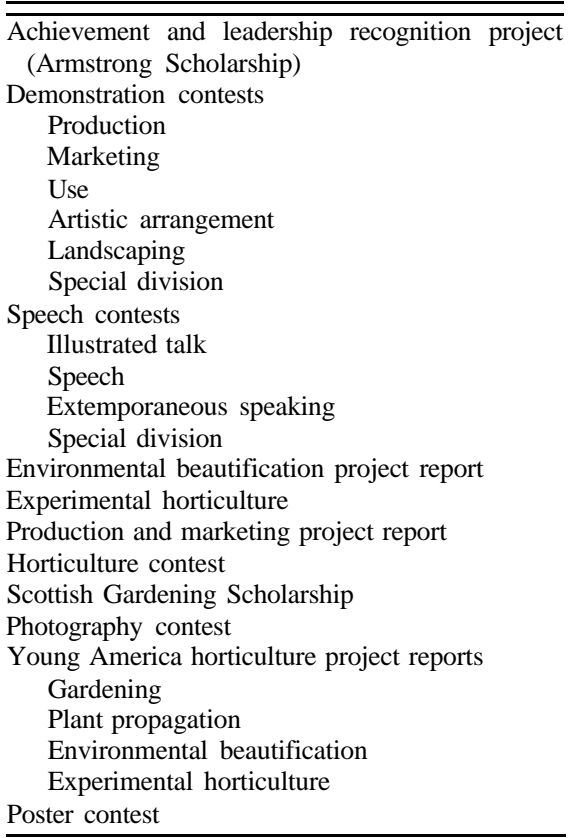

Each state is limited to one entry in each of the three divisions. Photographs must be entered through the NJHA state leader to prevent duplicate entries in a division. There are no subgroups based on age or affiliation with other youth organizations (Future Farmers of America, 4-H, etc.). Entries are judged "outstanding," "good," and "worthy." Participants between 8 and 22 years old may compete annually. They may enter more than one division per year but may not enter a division in which they have previously placed first. All photographs must have been taken during the previous year. Each entry must include a 35$\mathrm{mm}$ negative or slide, sample print(s), and completed entry form. Participants receive a certificate and enlarged mounted copy of their entry.

NJHA programs are designed to help youth obtain a basic understanding of horticulture and develop horticultural skills. Developing skills through participation in contests and related activities helps youth excel throughout life.

Information on this and other NJHA programs is available in the NJHA Project and Activities Guide for Contestants and Leaders. Single copies may be obtained at no charge from your NJHA state leader or from W.M.F.

\section{Literature Cited}

Fountain, W.M. 1991. The National Junior Horticultural Association: Serving youth and horticulture. HortScience 26:454,615.

Fountain, W.M. and R. Renner. 1992. The National Junior Horticultural Association-Developing horticultural skills and knowledge through the horticulture contest. HortScience 27:950, 1067.

William M. Fountain Dept. of Horticulture and Landscape Architecture Univ. of Kentucky Lexington, KY 40546-0091 